\title{
Eurotium cristatum, a new fungi probiotic from Fuzhuan brick tea, alleviated obesity in mice by modulating gut microbiota
}

\author{
Dingding Kanga , Meng Sua, Yanwen Duan*, a, b, c and Yong Huang ${ }^{*}, a, c$
}

aXiangya International Academy of Translational Medicine at Central South University, Changsha, Hunan 410013, China;

bHunan Engineering Research Center of Combinatorial Biosynthesis and Natural Product Drug Discovery, Changsha, Hunan 410011, China.

cNational Engineering Research Center of Combinatorial Biosynthesis for Drug Discovery, Changsha, Hunan 410011, China.

\section{Correspondence:}

*Email: Prof. Yong Huang, jonghuang@csu.edu.cn or Prof. Yanwen Duan,

ywduan66@sina.com. 


\section{Abstract}

Obesity is one of the major public health problems worldwide, mainly resulting from unhealthy lifestyles and diet. Gut microbiota dysbiosis may also lead to obese humans and animals. Modulating gut bacteria through fecal transplantation, the use of probiotics or certain dietary supplements, could normalize gut microbiota and subsequently alleviate obesity. Daily consumption of Fuzhuan brick tea (FBT) or its extracts have been observed to alleviate obesity in humans and experimental animals. In this study, high-fat diet-induced dysbiosis of gut microbiota in C57BL/6J mice was partially reversed by consumption of Eurotium cristatum, the dominant fungi during the manufacturing and storage of FBT. E. cristatum was able to modulate both gut fungi and bacteria composition, based on the analysis of microbiota composition of mice fecal samples. E. cristatum increased acetate and butyrate-producing bacteria in mice gut, and produced five times more butyrate than both obese and normal mice. Our results suggested that $E$. cristatum may be used as a fungi probiotic to beneficially modulate gut microbiota and to alleviate obesity in humans. 


\section{Introduction}

Obesity is a disease associated with numerous health problems, mainly caused by a shift in lifestyles towards less physical expenditure and more hyper-caloric foods ${ }^{1}$. It is closely linked with chronic and low-grade inflammation, which may cause insulin resistance, type 2 diabetes, fatty liver disease or cardiovascular disease ${ }^{2-4}$. Based on two recent population analysis from $1975-2016$ or $1980-2015$, about $10 \%$ of the world population, including over 100 million young people, were obese ${ }^{5}$. Obesity epidemic becomes a major threat to public health in modern human history ${ }^{6}$. Obesity treatment may include bariatric surgery or changing of lifestyles to increase exercise, which reduce calorie intake ${ }^{7}$. Antiobesity drugs, such as phentermine or orlistat, could suppress appetite or inhibit the absorption and utilization of fat ${ }^{8}$. Alternatively, regulating intestinal microbes has recently been used for the treatment of obesity, because microbiota dysbiosis plays prominent roles in the development of obesity ${ }^{9-12}$. A variety of probiotics, either individually or more often used as cocktails, were shown to alleviate obesity in a high-fat diet (HFD) fed rodents. For example, both Bifidobacterium and Lactobacillus were shown to attenuate HFD-induced obesity in animals and humans, including weight loss, reduced visceral fat and improved glucose tolerance ${ }^{13-15}$. Most of these bacteria produce short-chain fatty acids (SCFAs), such as acetate and butyrate, which reduce gut luminal $\mathrm{pH}$ and maintain an acidic environment ${ }^{16,17}$. Previous studies also highlighted the importance of SCFAs to improve chronic inflammatory diseases and to promote colonocyte health ${ }^{18}$. SCFAs were reported to suppress production of pro-inflammatory cytokines IL-1 $\beta$, enhance IL-10 expression and activate regulatory $T$ cells $\left(T_{\text {reg }}\right)$, to maintain intestinal homeostasis ${ }^{19}$. 
Humans have prepared and consumed a variety of fermentation products, such as pickles, sauerkraut, sourdough bread, artisanal cheeses, yogurts and kefir, craft beers and kombucha (fermented tea), in which various bacteria and fungi species are present, dated back to the seventh millennium before Christ ${ }^{20,21}$. Fermentation is very useful to preserve food and increase their flavor and nutrition ${ }^{22}$. Fuzhuan brick tea (FBT, also named Fu brick tea) is a fermented tea in the presence of a dominant fungi species named Eurotium cristatum, which is yellow-colored and is thus also called "golden flora" (“金花”) in Chinese ${ }^{23}$. Human consumption of FBT dated back to the $16^{\text {th }}$ century ${ }^{24}$, while recent pilot dietary interventions on humans in China have also shown that FBT consumption could improve metabolic disease conditions, which were summarized in Supplementary Table 1. FBT consumption or polysaccharides extracted from FBT were shown to reduce obesity, hyperlipidemia and arterial stiffening, as well as to improve metabolic syndrome in animals $^{25-28}$. The water or ethanol extracts of FBT reduced obesity through normalizing gut microbiota in mice 25,29 .

The dominant fungi E. cristatum in FBT play an important role in the flavor, color and health benefits of $\mathrm{FBT}^{30}$. We hypothesized that live E. cristatum in FBT may survive in the gastrointestinal tract during tea consumption. It might have served as a fungi probiotic to modulate gut microbiota, which led to the observed anti-obesity effects of FBT. In this study, we evaluated the effects of dietary supplementation of live E. cristatum to prevent dietinduced obesity in mice. Our study supports the use of E. cristatum as a fungi probiotic to modulate gut microbiota against obesity, which has been used by humans for about 600 years. 


\section{Results}

E. cristatum may survive in mouse intestine. In order to clarify if E. cristatum could survive during tea brewing and consumption, $10^{4}$ spores of $E$. cristatum isolated from FBT, were heated in water containing $20 \%(\mathrm{v} / \mathrm{v})$ glycerol at $50,70,80,85,90$ and $100{ }^{\circ} \mathrm{C}$ for 2 , 5 or 10 min, respectively (Supplemental Fig.1). E. cristatum survived even after 10 min in $90{ }^{\circ} \mathrm{C}$ and 2 min in $100{ }^{\circ} \mathrm{C}$. E. cristatum was next mixed with drinking water and fed to Kunming mice. E. cristatum could be isolated from mouse feces, even without feeding of E. cristatum for two weeks (Supplemental Fig. 2). There were no significant differences in body weight among mice fed with or without E. cristatum, as well as mice fed with the water extracts or filtered water extracts of Fuzhuan brick tea. These results suggested that $E$. cristatum from Fuzhuan brick tea is non-toxic and could survive in mice intestine.

E. cristatum reduced HFD-induced obesity in C57BL/6J mice. The HFD-induced C57BL/6J obese mouse model was used to explore if live E. cristatum may have any antiobesity effect, in comparison to mice that received normal chow diet (NCD). The high-fat diet mice that received FBT or filtered Fuzhuan brick tea (FFBT), in which live E. cristatum were removed by filtering the FBT extracts using a $0.22 \mu \mathrm{m}$ filter, were also used as controls (Fig. 1a-e). The mice in HFD group showed significant increases in body and liver weights, and epididymal fat accumulation, compared with mice in the NCD group (Fig. 1ae). In contrast, the high-fat diet mice that received E. cristatum $\left(10^{3} \mathrm{CFU} /\right.$ day) in drinking water showed significantly decreased body weight gain, liver weight and epididymal fat accumulation. The FFBT-treated mice also showed significant decrease in liver and 
epididymal fat, while FBT-treated mice only had statistically-insignificant lower body weight, in comparison to HFD-fed mice.
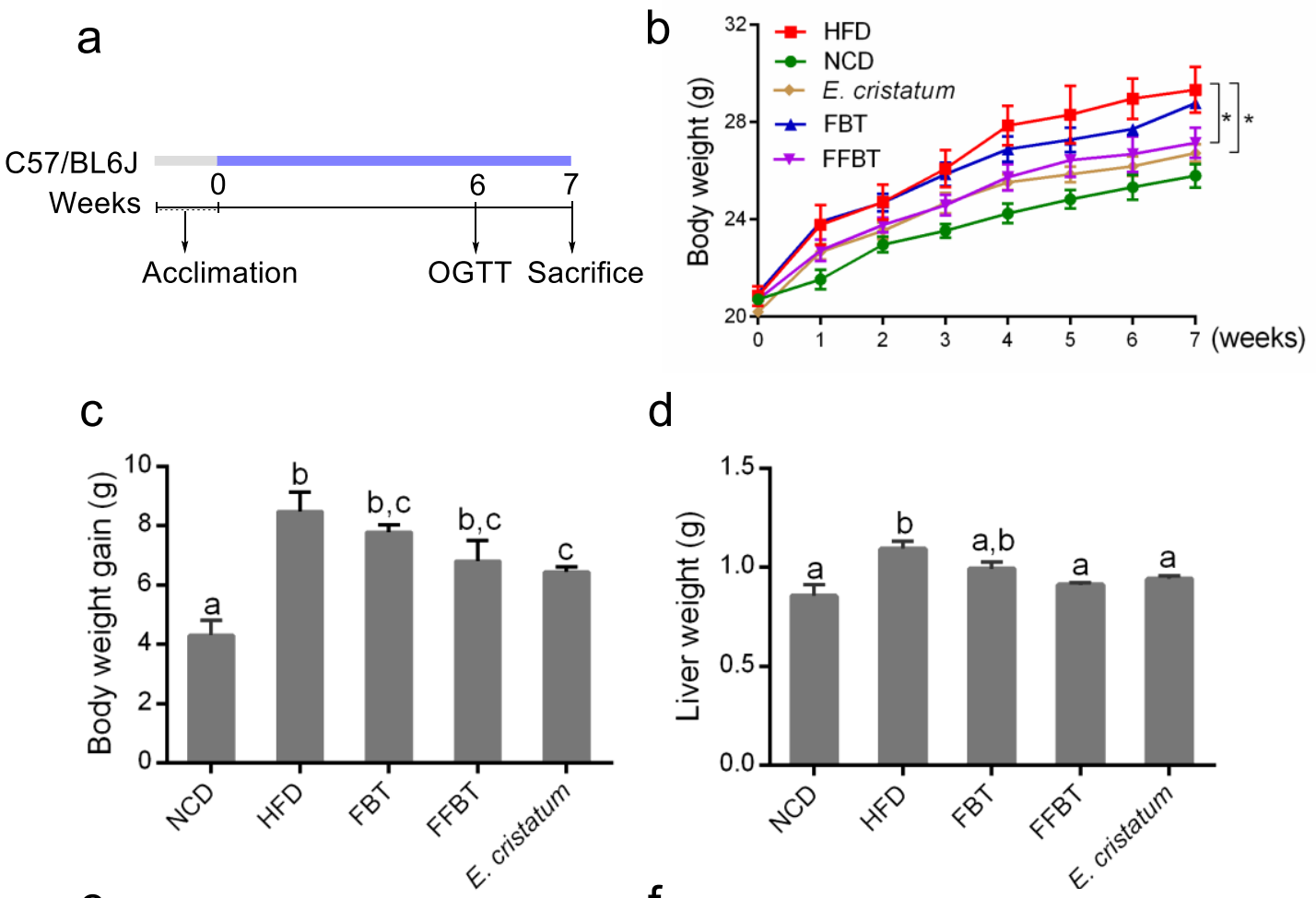

d
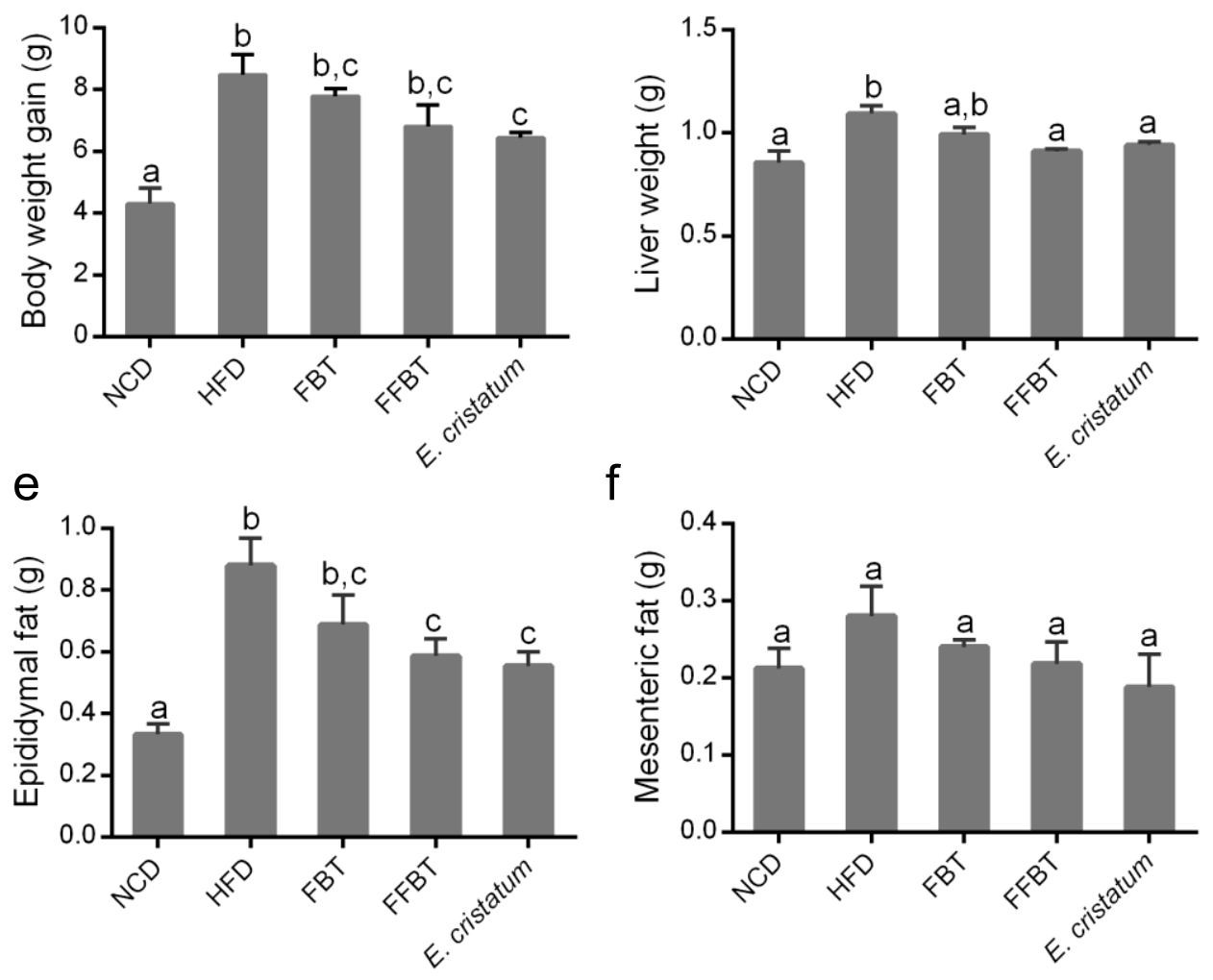

Fig. 1 | Dietary E. cristatum reduced body weight and fat accumulation in HFD-induced mice. a, The animal protocol. b, Effects of E. cristatum, FBT and FFBT on body weight in 7 weeks. c, Body weight gain. d, Liver weight. e, Epididymal and mesenteric fat. f, Data are expressed as mean \pm s.e.m. Body weight differences in $\mathbf{b}$ were analyzed using unpaired two-tailed Student's $t$-test $\left({ }^{*} P<0.05\right)$. Graph bars in figure $\mathbf{c}, \mathbf{d}, \mathbf{e}$ and $\mathbf{f}$ marked with different letters on top represent statistically significant results $(P<0.05)$ based on Newman-Keuls post hoc one-way ANOVA analysis, whereas bars labeled with the same letter correspond to results that show no statistically significant differences. In the case, whereas two letters are present on top of the bar, each letter should be compared separately with the letters of other bars to determine whether the results show statistically significant differences. 
E. cristatum increased glucose tolerance in HFD-fed mice. The effects of E. cristatum, FBT or FFBT consumption on glucose homeostasis of HFD-fed mice were next determined (Fig. 2a-c). Compared to HFD-fed mice that received only drinking water, the HFD-fed mice with daily consumption of $E$. cristatum, FBT or FFBT had reduced fasting blood glucose levels (FBG) and increased glucose tolerance. Since endotoxemia controls the production of pro-inflammatory cytokines in target tissues and may lead to chronic inflammation in HFD-fed mice ${ }^{31}$, the serum lipopolysaccharide (LPS) levels in these mice were further examined (Fig. 2d). Both FBT and FFBT consumption reduced the LPS level in HFD-fed mice, while E. cristatum had no significant effect.

E. cristatum reduced inflammation in HFD-fed mice. HFD-induced obese mice have been observed to produce higher levels of pro-inflammatory cytokines in hepatic tissues, including interleukin-1- $\beta$ (IL-1 $\beta$ ) and interleukin-6 (IL-6), while the level of the antiinflammatory cytokine interleukin (IL-10) was reduced in obese animals ${ }^{32}$. The IL-1 $\beta$ and IL-10 in hepatic tissues of the sacrificed mice were thus measured by enzyme-linked immune sorbent assay (ELISA) (Fig. 2e-f). The HFD-fed mice produced about $300 \mathrm{pg} / \mathrm{mL}$ $\mathrm{IL}-1 \beta$, while the consumption of $E$. cristatum, FBT or FFBT reduced the IL-1 $\beta$ level to about $200 \mathrm{pg} / \mathrm{mL}$, similar to the NCD-fed mice. Similarly, the levels of anti-inflammatory cytokine $\mathrm{IL}-10$ were also restored to those in NCD-mice through consumption of E. cristatum, FBT or FFBT. 
a

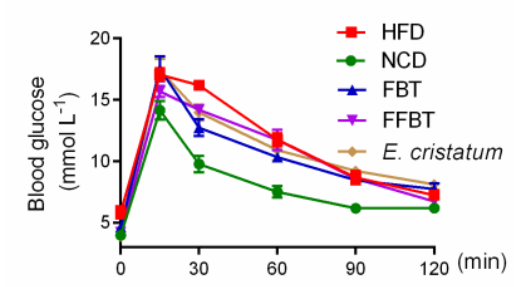

d

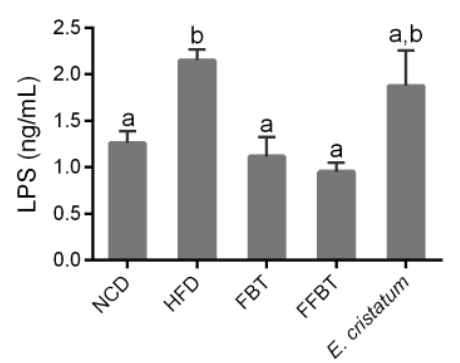

$\mathrm{b}$

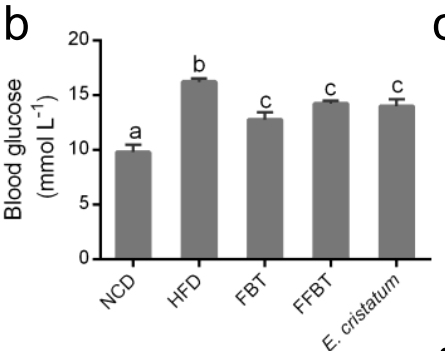

e

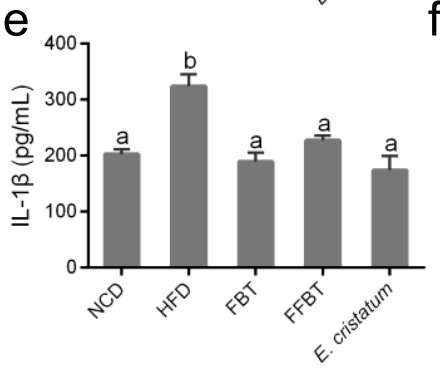

C

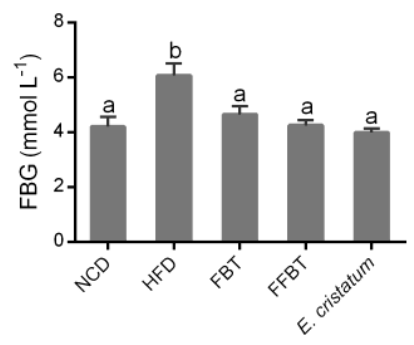

$f$

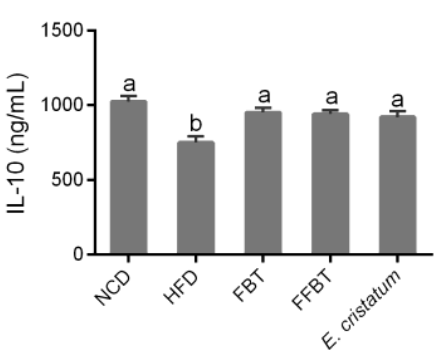

Fig. 2 | E. cristatum, FBT or FFBT reduced HFD-induced metabolic endotoxemia and systemic chronic low-grade inflammation, and improved glucose homeostasis. a, Effects of E. cristatum, FBT and FFBT on blood glucose using OGTT test. b. Blood glucose of $30 \mathrm{~min}$ of OGTT. c, Fasting blood glucose (FBG). d, LPS level. e, IL-1 1 . f, IL-10 in hepatic tissues. Data are expressed as mean \pm s.e.m. Graph bars in figure $\mathbf{b}, \mathbf{c}, \mathbf{d}, \mathbf{e}$ and $\mathbf{f}$ marked with different letters on top represent statistically significant results $(P<0.05)$ based on Newman-Keuls post hoc one-way ANOVA analysis.

E. cristatum altered the fungi diversity in mice gut. Since consumption of $E$. cristatum alone had significant anti-obesity effects of the HFD-fed mice (Fig. 1-2), we hypothesized that $E$. cristatum may be able to modulate mice gut microbiota through its interaction with commensal fungi and bacteria ${ }^{33,34}$. Therefore the composition, abundance and function of mice gut microbiota were next analyzed by high-throughput sequencing of the internal transcribed spacer 2 (ITS2) and 16S rRNA of caecal stool samples of the above C57BL/6J mice. After removing unqualified sequences, a total of 741,205 raw reads and an average of $37,060 \pm 23,543$ reads per mouse sample were obtained for ITS2 rDNA regions. Rarefaction of chao1 and observed OTUs indicated that the sequencing depth covered most of the fungi diversity, including rare new phylotypes (Supplemental Fig. 4a-b).

To evaluate fungi diversity in mice gut, the fungi $\beta$ diversity was measured. Constrained 
principal-coordinate analysis (CPCOA) based on the similarity index revealed distinct clustering of fungi composition for each mice group (Fig. 3a). E. cristatum- or FBT-treated HFD-fed mice were closer to NCD-fed mice. Further hierarchical clustering analysis did not reveal distinct separation of each mice group. Fungi taxonomic profiling in the genus level of intestinal fungi revealed that Euroteomycetes were more abundant in E. cristatumtreated mice than those of other groups, which suggested that E. cristatum had survived in mice intestine (Fig. 3c-d). Statistical analysis of metagenomic profiles (STAMP) of gut fungi also revealed several OTUs belonging to Scolecobasidium, Eurotium, Penicillium and Cosmospora, which were significantly altered in different mice groups (Fig. 3e-f). 
a

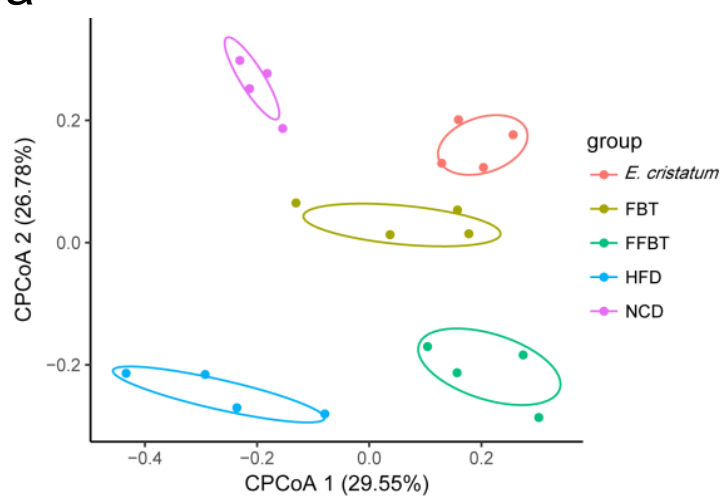

C

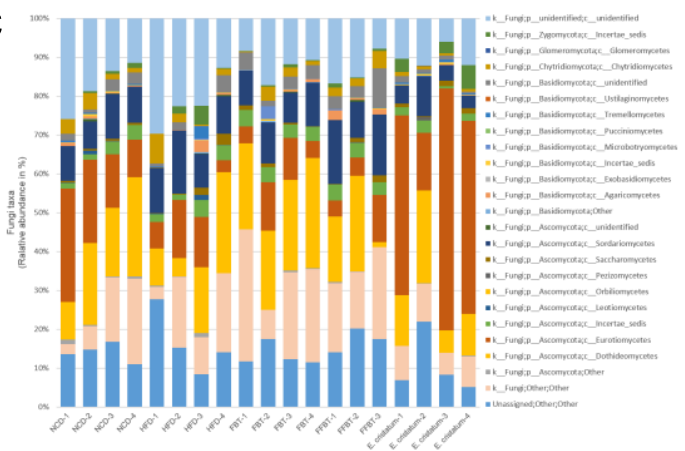

e
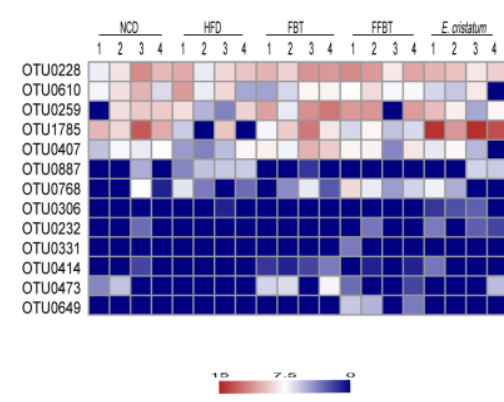

b

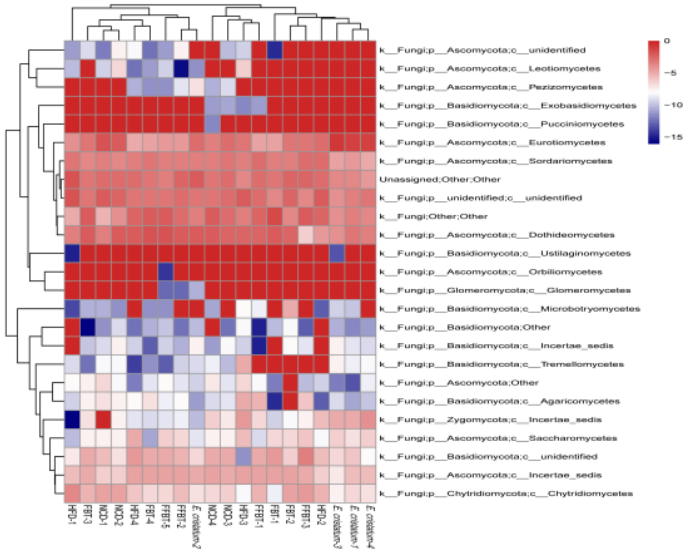

d

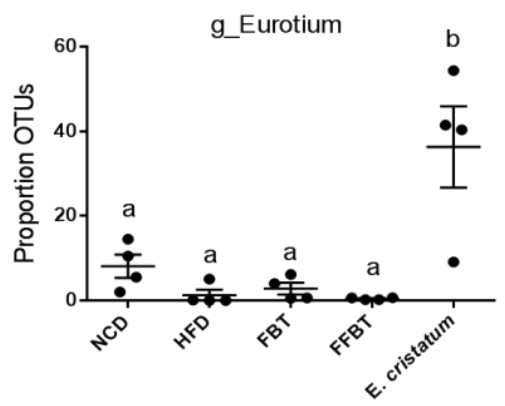

Fig. 3 | Dietary E. cristatum, FBT and FFBT beneficially altered the gut fungi composition. a, Constrained principal-coordinate analysis based on the cao similarity index with the PERMANOVA significance test of the ITS2 region. b. Hierarchical clustering showing the relative abundance of representative OTUs. c, Fungi taxonomic profiling in the family level of intestinal fungi from different mice groups. $\mathbf{d}$, The relative abundances of $E$. cristatum. e, The abundance of OTUs significantly altered by $E$. cristatum, FBT and FFBT in HFD-fed mice based on STAMP. f, Represented bacterial taxa information (species, genus, family, and phylum) of OTUs from e.

Dietary E. cristatum beneficially altered the gut bacteria. The V4 region of $16 \mathrm{~S}$ rRNA of mice gut bacteria were also obtained, which included a total of 765,760 raw reads and an average of $30,630 \pm 17,252$ reads per mouse sample. The rarefaction curves analysis of chao1 and observed OTUs revealed that most of the commensal bacteria diversity were 
covered (Supplemental Fig. 4). The $\beta$ diversity of the gut bacteria was also evaluated using CPCoA-based Bray-Curtis similarity index, which revealed distinct clustering of bacteria composition for each mice group (Fig. 4a). Compared with other groups, E. cristatumtreated HFD-fed mice were closer to NCD-fed mice in both primary and secondary ordination axes. The inter-sample correlation calculated by Pearson coefficient analysis revealed that the gut bacteria of NCD and E. cristatum groups were more closely related (Fig. 4b).

Bacterial taxonomic profiling in the phylum level of intestinal bacteria from different mice groups were next analyzed (Fig. 4c). The gut microbiota of HFD-fed mice were characterized by an increased Firmicutes-to-Bacteroidetes ratio $(F / B)$, which was consistent the previous reports ${ }^{35,36}$. Notably, the treatment of FBT in HFD-fed mice reversed the F/B ratio, while FFBT and E. cristatum intervention exhibited limited effects on the relative abundances of Firmicutes and Bacteroidetes and the ratio of F/B (Fig. 4d).

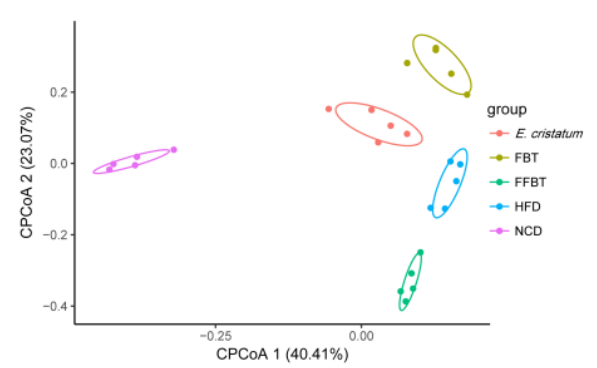

C

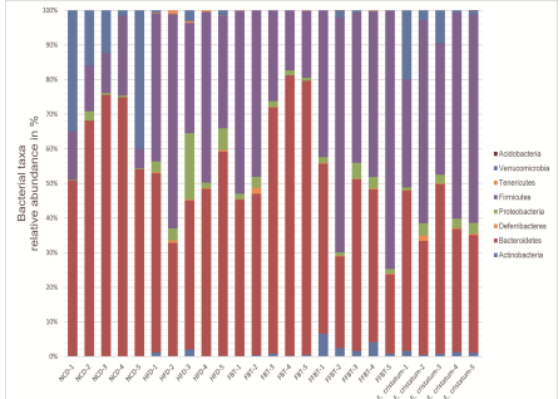

b

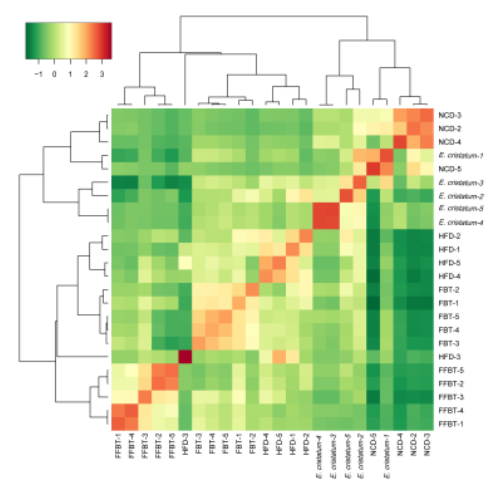

d

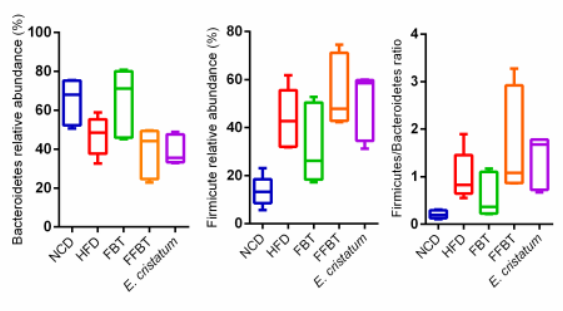

Fig. 4 | Dietary E. cristatum, FBT and FFBT beneficially altered the gut bacterial composition. a, 
Constrained Principal-coordinate analysis based on the Bray-Curtis similarity index with the PERMANOVA significance test about 16S rRNA. b, Inter-sample correlation calculated by the Pearson coefficient. c, Bacterial taxonomic profiling in the phylum level of intestinal bacteria from different mice groups. d, The relative abundances of Bacteroidetes, Firmicutes, and F/B. e, The abundance of OTUs significantly altered by E. cristatum, FBT and FFBT in HFD-fed mice based on STAMP. f, Represented bacterial taxa information (species, genus, family, and phylum) of OTUs from e.

STAMP was used to identify the specific bacterial phylotypes of each mouse. Compared with NCD-fed mice, there were 132 bacterial OTUs were significantly altered in HFD-fed mice, in which 118 OTUs increased and 14 OTUs decreased. For HFD-fed mice treated with FBT, FFBT or E. cristatum, there were 67 (18 increased and 49 decreased), 96 (26 increased and 66 decreased), 85 (36 increased and 49 decreased) altered OTUs, respectively, compared with HFD-fed mice. Interestingly, 43, 51 and 45 bacterial OTUs in groups of $E$.cristatum, FBT and FFBT, were altered towards NCD-fed mice, respectively. Analysis of the gut bacteria altered by E. cristatum consumption indicated that SCFAsproducing bacteria Lactobacillus increased over 14 folds, in comparison to HFD-fed mice (Fig. 4e). Bifidobacterium, the other SCFAs-producing bacteria, increased dramatically. In contrast, Odoribacteracae ${ }^{37}$, Parabacterioide $^{12}$, Sutterella ${ }^{38}$ and Clostridium, decreased in HFD-fed mice, which were negatively correlated with diet-induced obesity. These results suggested that E. cristatum, as well as FBT and FFBT, may modulate the gut bacteria in HFD-fed mice and resulted in a microbiota composition close to that of NCD-fed mice.

PICRUSt analysis of the potential function of gut microbiota in different groups. In order to study the potential function of gut microbiota in different groups, linear discriminant analysis effect size (LEfSe) was used to infer the relative abundance of Kyoto Encyclopedia of Genes and Genomes (KEGG) pathways, which were predicted by 

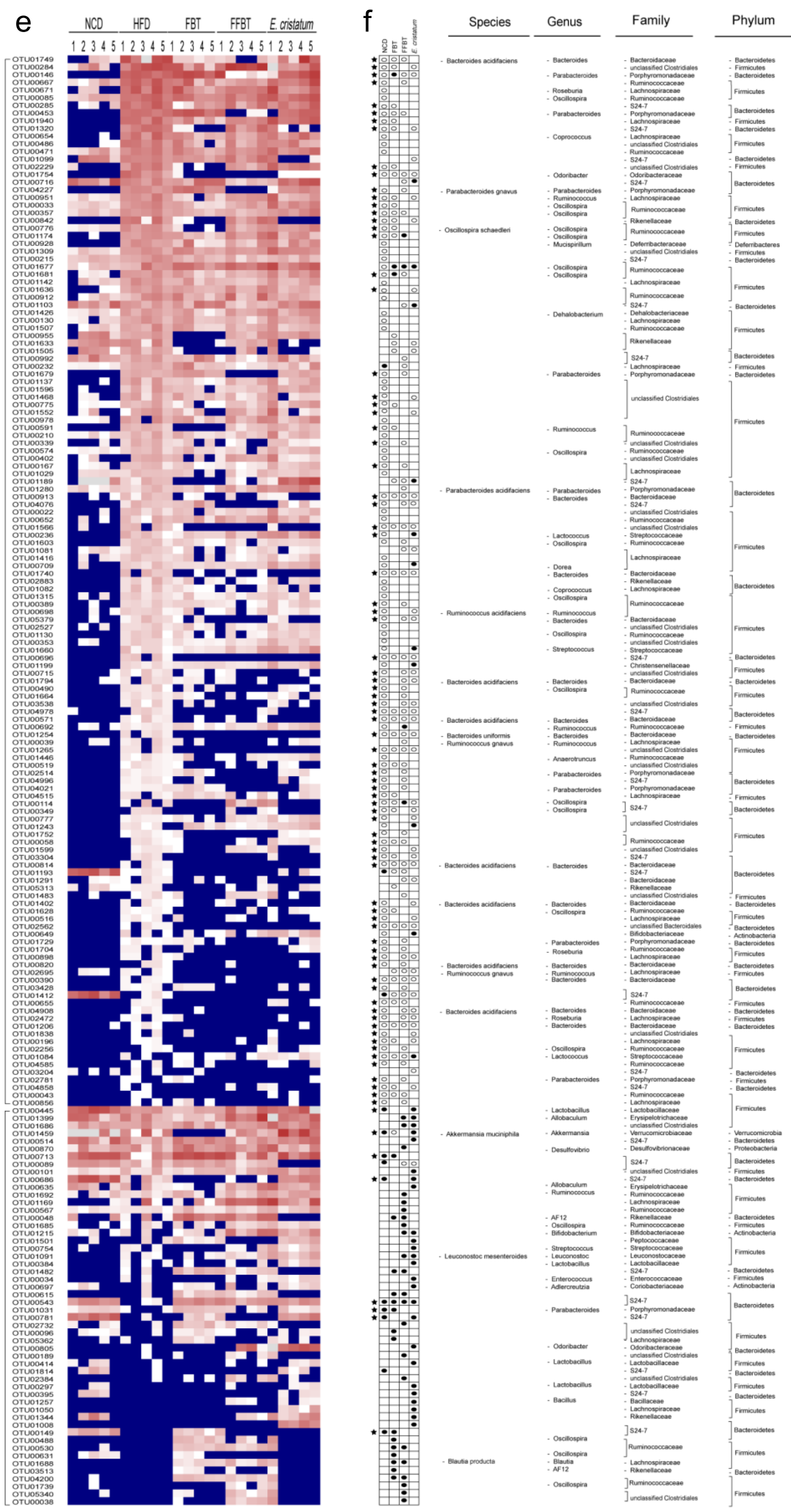

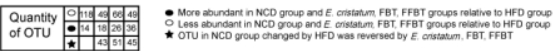

Fig. 4 | Continued 
phylogenetic reconstruction of unobserved states (PICRUSt) ${ }^{39}$. The HFD-fed mice had a reduced capacity for energy metabolism and increased capacity of carbohydrate metabolism, consistent with a recent metagenomics-based study ${ }^{35}$. Notably, both galactose metabolism and starch metabolism were significantly lower in E. cristatumtreated mice than in those HFD-fed mice. In addition, the energy metabolic pathways, including fatty acid and nucleotide metabolisms were significantly lower in HFD-fed mice and they could be reversed by E. cristatum consumption. These metabolic changes could be also identified in the FBT and FFBT groups (Supplemental Fig. 5a-c).

E. cristatum increased SCFAs production in mice gut. The SCFAs analysis of mice fecal samples revealed dramatic differences among these mice groups (Fig. 5). Comparing to NCD-fed mice, the HFD-fed mice showed decreased levels of acetate and propionate in their feces samples, while they had a comparable level of butyrate production. In contrast, consumption of $E$. cristatum in HFD-fed mice significantly increased the amount of acetate and butyrate. Notably, the amount of butyrate in the E. cristatum group increased over five times over both NCD-fed and HFD-fed mice. The amount of butyrate in the mice fecal samples in FBT mice group also increased significantly. The increasing amount of acetate and butyrate in E. cristatum-treated mice was consistent with the increased acetate- and butyrate-producing bacteria in mice gut, such as Bifidobacterium and Lactobacillus (Fig. 34).

Determination of the optimal dosage of E. cristatum to reduce HFD-induced obesity in C57BL/6J mice. Three different dosages of E. cristatum, $10^{4} \mathrm{CFU} /$ day, $10^{3} \mathrm{CFU} /$ day and $10^{2} \mathrm{CFU} /$ day, were employed to HFD-induced C57BL/6J mice for 8 weeks. 

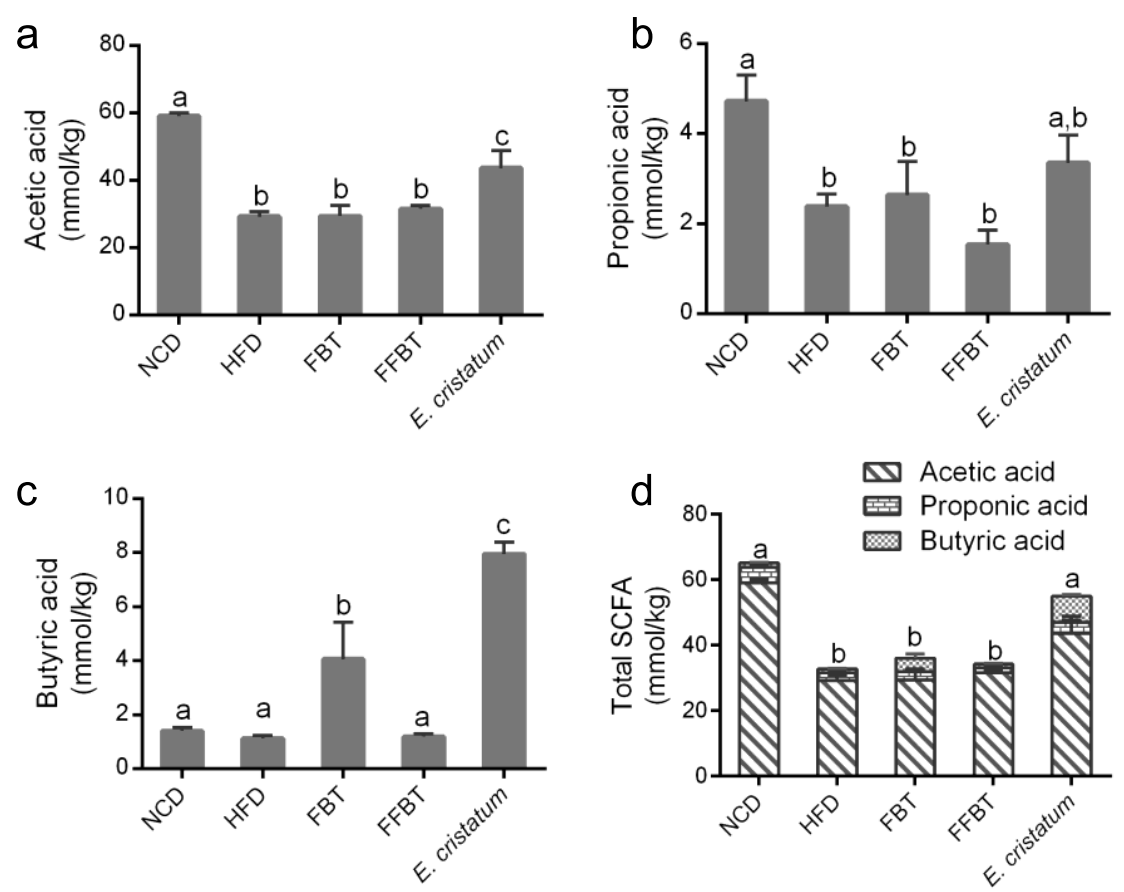

Fig. 5 | Effects of E. cristatum, FBT and FFBT on SCFAs production in mice gut. a, Effects of $E$. cristatum, FBT and FFBT on acetic acid, b, Propionic acid, c, Butyric acid and d, Total SCFAs. Data are expressed as mean \pm s.e.m. Graph bars in $\mathbf{a}, \mathbf{b}, \mathbf{c}$ and $\mathbf{d}$ marked with different letters on top represent statistically significant results $(P<0.05)$ based on Newman-Keuls post hoc one-way ANOVA analysis.

Alternatively, E. cristatum ( $10^{3} \mathrm{CFU} /$ day) were given to HFD-induced mice only for 3 and 5 weeks, respectively. Consumption of different dosages or various periods of E. cristatum, all decreased the body weight gain, liver weight and fat accumulation in HFD-fed mice (Fig. 6). The HFD-fed mice receiving E. cristatum $\left(10^{3} \mathrm{CFU} /\right.$ day) for 8 weeks gained slightly less weights than other groups. The E. cristatum $\left(10^{3} \mathrm{CFU} /\right.$ day, 8 weeks) group also had better blood glucose tolerance than other mice groups, while higher or lower dosage of $E$. cristatum ( $10^{2}$ or $10^{4} \mathrm{CFU} /$ day, 8 weeks) were less effective to improve blood glucose homeostasis of HFD-fed mice, indicated by the OGTT test (Fig. 7). Consumption of $E$. cristatum for 3 or 5 weeks also increased glucose tolerance in HFD-fed mice, which suggested that short-term intervention of the microbiota dysbiosis of HFD-fed mice using E. cristatum might also be effective (Fig. 7c-d). 

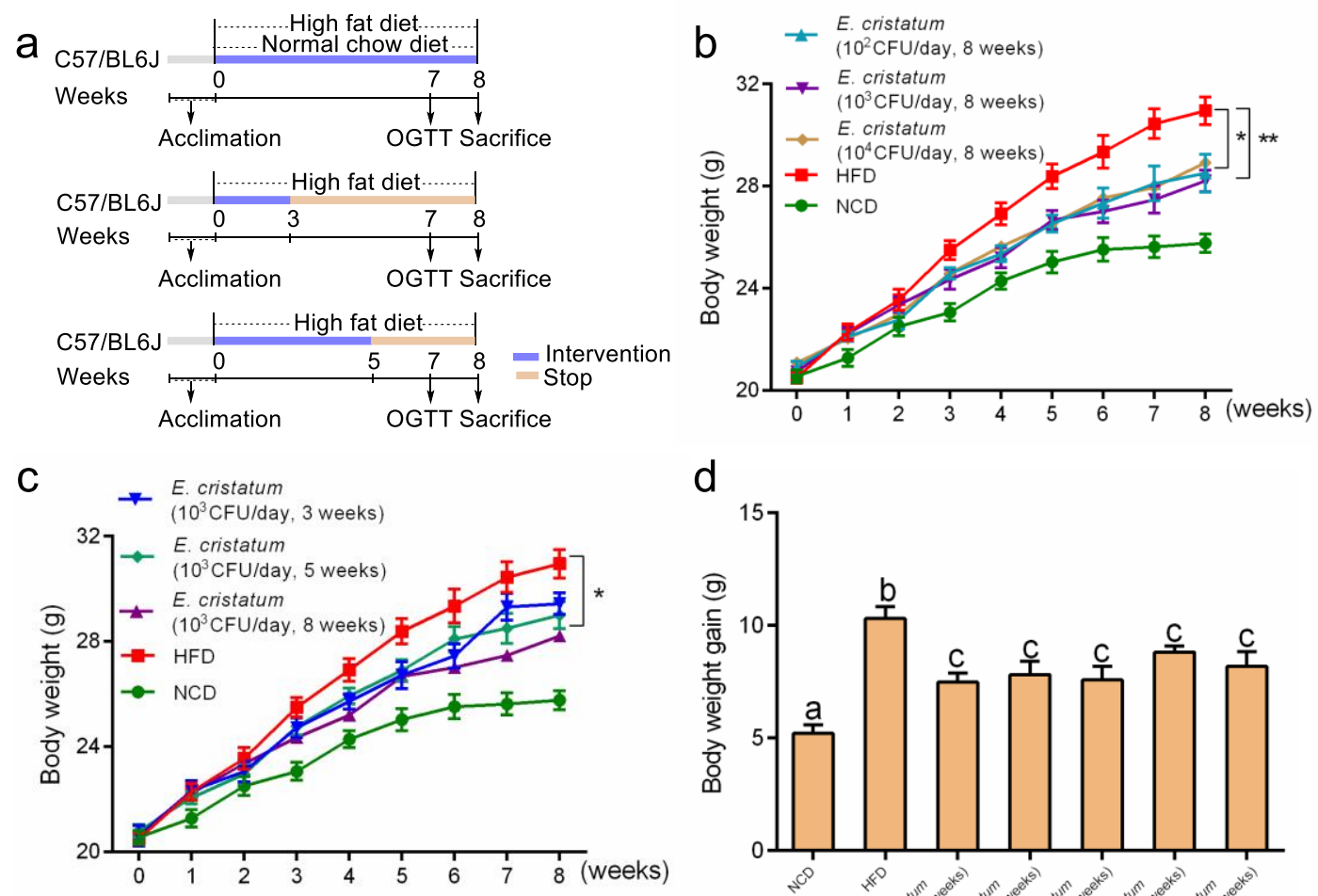

e

f
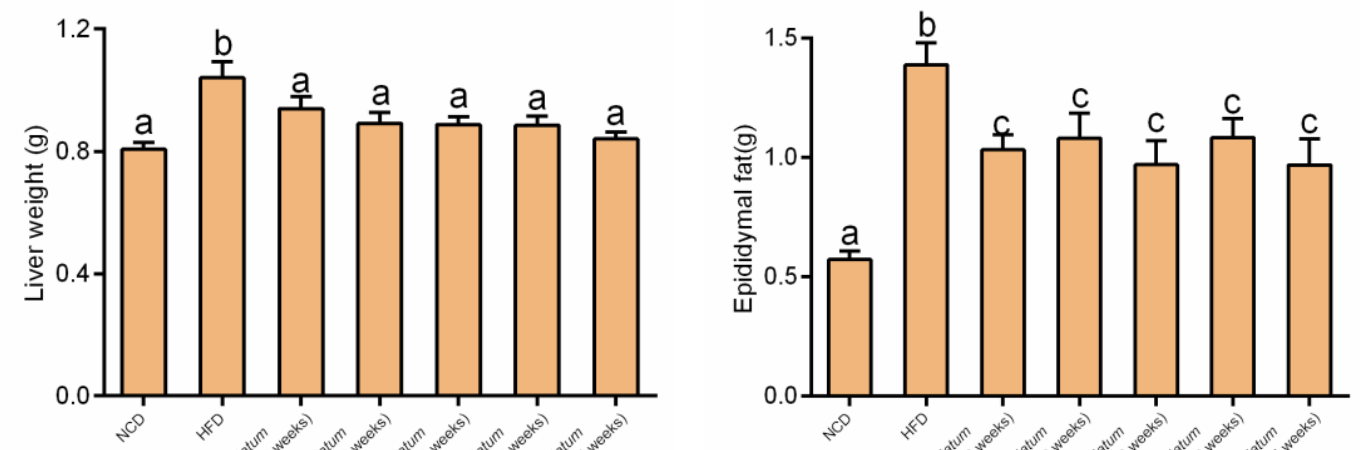

g

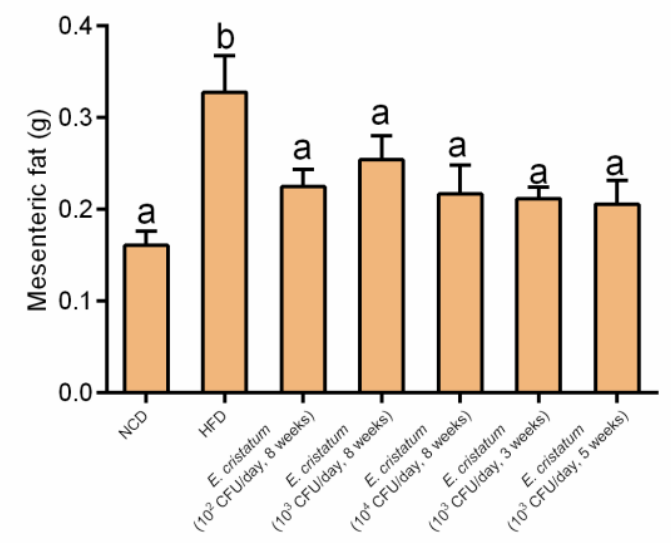

Fig. 6 | Dietary E. cristatum reduced body weight and fat accumulation in HFD-induced mice. a, Animal protocol. $\mathbf{b}$, Effects of different dosage of E. cristatum on body weight of eight weeks. c, Effects of 
consumption various time of $E$. cristatum on body weight of eight weeks. d, Body weight gain. e, Liver weight. f, Epididymal fat. $\mathbf{g}$, Mesenteric fat. Data are expressed as mean \pm s.e.m. Body weight differences in b, $\mathbf{c}$ were analyzed using unpaired two-tailed Student's $t$-test $\left({ }^{*} P<0.05,{ }^{*} P<0.01\right)$. Graph bars in figure $\mathbf{d}, \mathbf{e}, \mathbf{f}$ and $\mathbf{g}$ marked with different letters on top represent statistically significant results $(P<0.05)$ based on Newman-Keuls post hoc one-way ANOVA analysis.
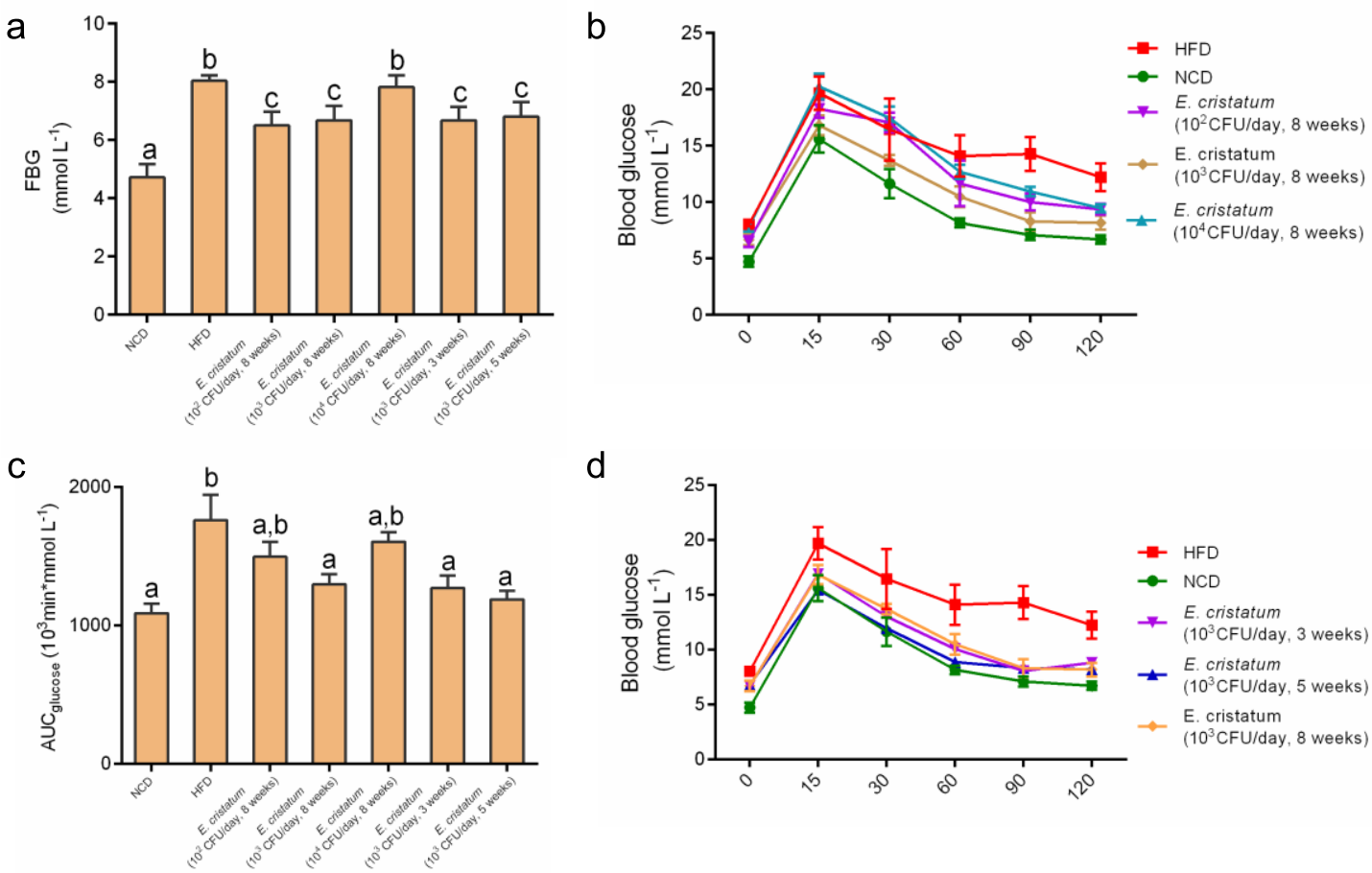

Fig. 7 | Dietary E. cristatum improved glucose homeostasis. a, Fasting blood glucose. b, Effects of different dosage of E. cristatum on blood glucose of OGTT. c, Glucose area under the curve (AUC glucose) during OGTT. d, Effects of consumption various time of E. cristatum on blood glucose of OGTT. Data are expressed as means \pm s.e.m. Graph bars in a and $\mathbf{c}$ marked with different letters on top represent statistically significant results $(P<0.05)$ based on Newman-Keuls post hoc one-way ANOVA analysis.

\section{Discussion}

One of the hallmarks of obesity is the dysbiosis of gut microbiota, and normalization of the gut microbiota through fecal transplantation, consumption of dietary fibers and consumption of certain probiotics may be instrumental to manage the obesity epidemic in the world. In the current study, we hypothesized that E. cristatum, derived from Fuzhuan brick tea, may be adopted as a promising fungi probiotic against obesity. In the HFD-fed mice model, E. cristatum was shown to alleviate HFD-induced mice obesity, by reducing 
inflammation and improving their glucose homeostasis. The normalization of gut microbiota and the increased production of butyrate in E. cristatum-treated obese mice contributed to the observed anti-obesity effect (Fig. 8). Fuzhuan brick tea extracts and polysaccharides have previously been shown to alleviate HFD-induced mice obesity, probably also through modulation of gut microbiota $25,26,29$. Our study is consistent with these previous studies, in which the FFBT-treated, HFD-fed mice showed reduced liver weight and epididymal fat, and both FBT- and FFBT-treated mice groups showed improved glucose homeostasis and attenuated inflammation, compared to HFD-fed mice.

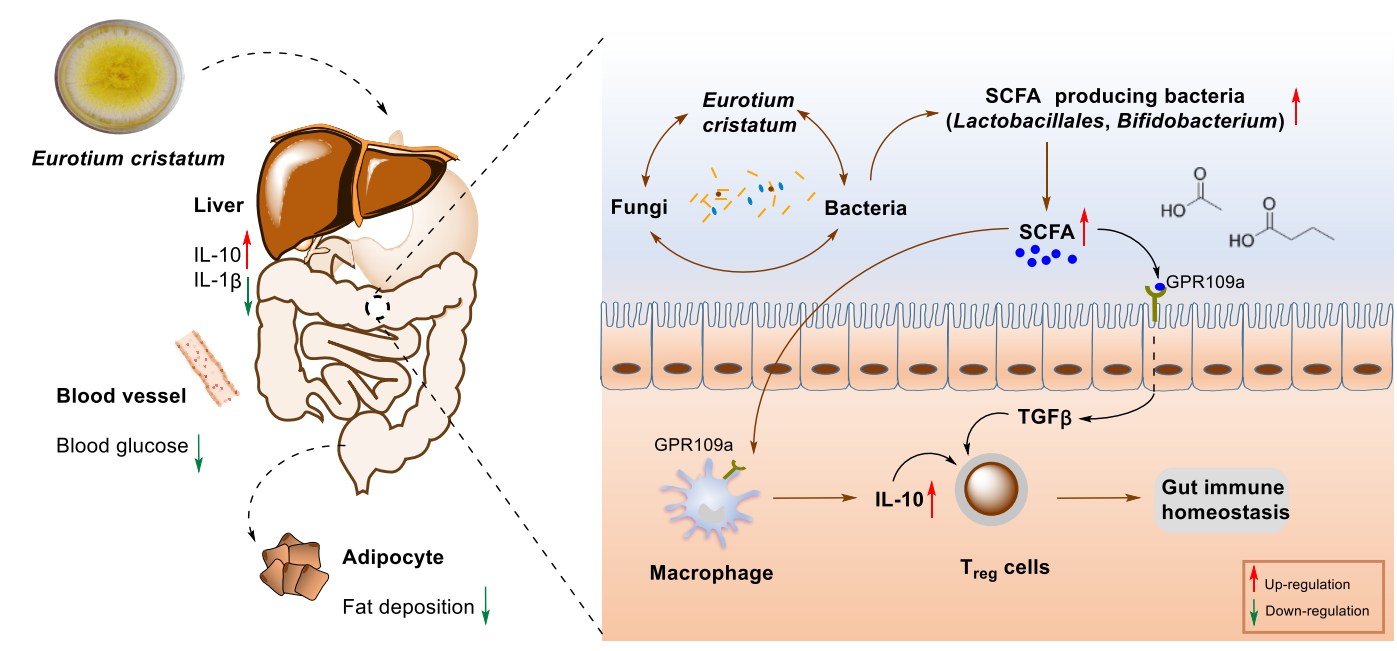

Fig.8 | The proposed mechanism of dietary E. cristatum against HFD-induced obesity in mice. The interactions between dietary $E$. cristatum and gut microbiome may lead to the normalization of microbiota dysbiosis in the gut of HFD-fed mice. The increase of SCFAs-producing bacteria, including Bifidobacterium and Lactobacillus, leads to the production of more SCFAs in mice gut, especially acetate and butyrate. These changes lowered inflammatory cytokines and led to the observed anti-obese effects.

The gut microbiota of obese humans and animals have decreased ratio of Bacteroidesto-Firmicutes, and Firmicutes are crucial in obesity-related metabolic disorder ${ }^{41}$. Therefore the treatment of obese mice with Myrciaria $d u b i a^{42}$, melatonin ${ }^{43}$ and other probiotics reversed this ratio and alleviated obesity. The consumption of FBT in HFD-fed mice could restore this lopsided microbiota, while supplementation of FFBT and E. cristatum were 
unable to reverse this unbalance. Probiotic bacteria, such as SCFAs-producing Lactobacillus, Bifidobacterium and Leuconostoc were shown previously to prevent or treat gastrointestinal disorders ${ }^{44-46}$, and to have anti-obesogenic or anti-diabetic potential ${ }^{47-49}$. Our study demonstrated that E. cristatum enhanced Lactobacillus, Bifidobacterium and leuconostoc in mice gut. Akkermansia muciniphila were shown to tighten gut barrier and reversed high-fat diet-induced obesity and improve glucose tolerance under high-fat diet conditions in conventional mice ${ }^{15,50,51}$. A. muciniphila in the E. cristatum-treated HFD-fed mice group also increased slightly. These results suggested that E. cristatum could modulate gut microbiota by increasing beneficial bacteria in mice gut. Although there is a strong correlation between gut fungi and host health, few studies were reported to manage metabolic disorders induced by high-fat diet by regulating gut fungi ${ }^{33,34}$. Our study also demonstrated that $E$. cristatum were able to modulate both fungi and bacteria composition (Fig. 3-4).

SCFAs, generated by the fermentation of intestinal microbiota ${ }^{52,53,54}$, are important to control chronic inflammatory diseases ${ }^{55,56}$ and maintain gut homeostasis ${ }^{57}$. The generation of a large amount of butyrate in the gut of E. cristatum-treated HFD-fed mice was unprecedented, which might contribute to the observed anti-obesity effects. Locally produced butyrate played essential role for the development of intestinal and systemic immune system. For example, butyrate may directly or indirectly induce $\mathrm{T}_{\text {reg }}$ differentiation, including IL-10 producing $T_{\text {reg }}$ cells. It was consistent with the increased level of IL-10, and the decreased level of IL-1 $\beta$ in E. cristatum-treated HFD-fed mice (Fig. 2). Similar effects were also observed in FBT-treated HFD-fed mice. 
In conclusion, our study strongly suggested that $E$. cristatum had significant anti-obesity effects in HFD-fed mice by modulating gut microbiota. Therefore, it may be used as a fungi probiotic to alleviate obesity, or other metabolic diseases, such as type II diabetes involving microbiota dysbiosis. Although Fuzhuan brick tea has already been consumed by humans for hundreds of years, the potential toxicity of the direct consumption of E. cristatum in humans needs to be established (Fig. S1). Since low dosage and short period of $E$. cristatum consumption may also have certain anti-obesity effects in HFD-fed mice (Fig. 7), E. cristatum may be used as a promising probiotic to alleviate obesity epidemic in the near future.

\section{Methods}

Preparation of E. cristatum, FBT and FFBT. E. cristatum CB10001 was isolated from Fuzhuan brick tea (FBT) (Jiuyang Processing Factory Co., Ltd., Yiyang, Hunan, China), which was confirmed to be E. cristatum based on its $18 \mathrm{~S}$ rRNA sequence. E. cristatum CB10001 was grown in M40Y medium and its spores were harvested and stored in $20 \%$ glycerol and kept in $-80^{\circ} \mathrm{C}$. The extracts for Fuzhuan brick tea (FBT) were prepared by brewing the tea with $85^{\circ} \mathrm{C}$ deionized $\mathrm{H}_{2} \mathrm{O}$, which was previously boiled. Briefly, Fuzhuan brick tea $(8 \mathrm{~g})$ was brewed in $1 \mathrm{~L}$ deionized $\mathrm{H}_{2} \mathrm{O}\left(85^{\circ} \mathrm{C}\right)$ in a household teapot for $15 \mathrm{~min}$. The filtered Fuzhuan brick tea (FFBT) was prepared by filtering the prepared FBT through a defecator $(0.22 \mu \mathrm{m}$, thermo, USA).

Survival of E. cristatum in elevated temperature. E. cristatum $\left(10^{4} \mathrm{CFU}\right)$ were suspended in $1 \mathrm{~mL} 20 \%$ glycerol and heated for $2 \mathrm{~min}, 5 \mathrm{~min}, 10 \mathrm{~min}$ under 50, 70, 80, 85, 
90 and $100{ }^{\circ} \mathrm{C}$, respectively. Then E. cristatum in each sample was serially diluted and spread onto M40Y agar plate. The plates were incubated at $30^{\circ} \mathrm{C}$ for $48 \mathrm{~h}$, and the resulting colonies were counted.

Dosage. Typically an adult $(60 \mathrm{~kg})$ may consume $10 \mathrm{~g}$ of Fuzhuan brick tea per day. Therefore the dosage of a mouse to consume the Fuzhuan brick tea was set as $1.6 \mathrm{mg} / \mathrm{g}$ $(1.6 \mathrm{mg} / \mathrm{g}=10 \mathrm{~g} / 60 \mathrm{~kg} \times 10)$ Fuzhuan brick tea per gram of individual mouse weight, which was about 10 times of Fuzhuan brick tea a human might have consumed. Since Fuzhuan brick tea contains $E$. cristatum $\left(2 \times 10^{5} \mathrm{CFU} / \mathrm{g}\right)$, the dosage of $E$. cristatum per mouse was set at $10^{3} \mathrm{CFU} /$ day first. To optimize the dosage of E. cristatum, $10^{2}$ or $10^{4} \mathrm{CFU} /$ day/mouse of E. cristatum were also used.

Animals. All animals in this study were purchased from Hunan Silaikejingda Experimental Animal Company Limited (Hunan, China). They were bred in the specific pathogen-free animal (SPF) facility of the Department of Laboratory Animals at Central South University (Changsha, Hunan, China) and kept under controlled light conditions (12 h light-dark cycle), with free access to water and diet.

Kunming mice (4-week, male) were randomly distributed into five groups, and each group had five mice. They had free access to chow diet containing $13.5 \%$ fat. The mice in normal chow diet group (NCD) had free access to drinking water. The mice in E. cristatum groups (10 $\mathrm{CFU} /$ day) or $\left(10^{3} \mathrm{CFU} /\right.$ day) only had access to drinking water containing different amount of E. cristatum. The mice in the Fuzhuan brick tea group (FBT) only had access to FBT as drinking water, while the mice in the filtered Fuzhuan brick tea group (FFBT) only had access to FFBT as drinking water. All the treatment groups, including E. cristatum (10 
CFU/day), E. cristatum ( $10^{3} \mathrm{CFU} /$ day), FBT and FFBT were given at every other 4-weeks, until the end of $16^{\text {th }}$ week. The body weight of individual mouse was measured every other week and the feces of the mice were also collected every other week.

C57BL/6J mice (6-week, male) were randomly distributed into five groups, and each group contained five mice. They had free access to chow diet (13.5\% fat) or high-fat diet $(60 \%$ fat) (Trophic Animal Feed High-Tech Co. LTD, Jiangshu, China). E. cristatum ( $10^{3} \mathrm{CFU} /$ day), FBT and FFBT were given to HFD-fed mice in drinking water for 7 weeks.

Optimal dosage of E. cristatum. To identify the optimal dosage of E. cristatum, C57BL/6J mice (6-week, male) were randomly divided into 7 groups and each group had 10 mice. They had free access to chow diet (13.5\% fat) or high-fat diet ( $60 \%$ fat). The 7 groups included: normal chow diet group (NCD), high-fat diet group (HFD), high dosage of $E$. cristatum group ( $10^{4} \mathrm{CFU} /$ day, 8 weeks $)$, middle dosage of E. cristatum group $\left(10^{3} \mathrm{CFU} /\right.$ day, 8 weeks) and low dosage of $E$. cristatum group ( $10^{2} \mathrm{CFU} /$ day, 8 weeks), as well as $E$. cristatum group ( $10^{3} \mathrm{CFU} /$ day, 3 weeks $)$ and E. cristatum group $\left(10^{3} \mathrm{CFU} /\right.$ day, 5 weeks), in which the HFD-fed mice were given E. cristatum for only 3 or 5 weeks, respectively.

Isolation and identification of E. cristatum from Kunming mice. The feces of Kunming mice (200 mg) were suspended in $1 \mathrm{~mL}$ sterile water. Then $100 \mu \mathrm{L}$ supernatant was spread onto M40Y agar plates and cultured at $30^{\circ} \mathrm{C}$ for $48 \mathrm{~h}$. The $18 \mathrm{~S}$ rDNA were PCR amplified from the isolated strain. The phylogenetic analysis of the 18S rRNA was performed using Mega 6 using the Neighbor-Joining method.

Oral glucose tolerance test. Overnight-fasted mice were administered a $2 \mathrm{~g} \mathrm{~kg}^{-1}$ OGTT ( $20 \%$ glucose solution) by oral gavage. Their blood glucose was measured by tail bleeding 
at time $0,15,30,60,90$ and 120 min after oral gavage, using a glucose meter (Sannuo Biosensor Co., Ltd, Hunan, China).

Cytokine measurements. IL-1 $1 \beta$, IL-10 protein levels were measured using commercial ELISA kits (eBioscience, USA).

Gut microbiota analysis. Mice feces were stored at $-80^{\circ} \mathrm{C}$, and the total DNAs were extracted using a stool DNA extraction kit (Omega, USA). For each caecal stool sample, the $16 \mathrm{~S}$ rRNA gene comprising $\mathrm{V} 4$ regions were amplified, using a forward primer $515 \mathrm{~F}$ (5'-GTGCCAGCMGCCGCGGTAA-3') and a reverse primer 806R (5'GGACTACHVGGGTWTCTAAT-3'). Both primers also contained a unique 12-base barcode to tag each PCR product. The ITS2 region of fungi in the caecal stool samples were amplied using a forward primer ITS7F (5'-GTGARTCATCGARTCTTTG-3') and a reverse primer ITS4R (5'-TCCTCCGCTTATTGATATGC-3'), which also contained a unique 12-base barcode to tag each PCR product. The high throughput sequencing was carried out using the Illumina MiSeq platform to generate $2 \times 250$ bp paired-end reads. The raw reads were quality filtered. The primers and barcode in each read were trimmed using QIIME $^{58}$ software package. The resulting sequences were then assigned to operational taxonomic units (OTUs) with $97 \%$ identity, followed by the selection of representative sequences.

The alpha and beta diversity were determined using a UniFrac analysis and a constrained principal coordinate analysis ( $\mathrm{CPCOA})$. The taxonomic abundance and characterize differences between groups were estimated by Linear discriminant analysis of the effect size (LEfSe). Phylogenetic investigation of communities by reconstruction of unobserved 
states (PICRUSt) ${ }^{39}$ was performed to identify functional genes in the sampled microbial community on the basis of the data in the KEGG pathway database.

SCFA analysis. SCFAs were similarly analyzed as described before ${ }^{59}$. Briefly, $50 \mathrm{mg}$ fecal sample was blended with $500 \mu \mathrm{L}$ acetonitrile and acidified with $5 \%(\mathrm{v} / \mathrm{v}) \mathrm{H}_{2} \mathrm{SO}_{4}$. Then the mixture was ultrasonically extracted for $10 \mathrm{~min}$ and centrifuged at $15000 \mathrm{rpm}$ for $10 \mathrm{~min}$. The supernatant was filtered through a $0.22 \mu \mathrm{m}$ filter. SCFAs were measured using a gas chromatograph-mass spectrometer (GCMS-QP2010 Ultra system, Shimadzu Corporation, Japan), which was equipped with an Rtx-Wax column $(30 \mathrm{~m} \times 0.25 \mu \mathrm{m} \times 0.25 \mu \mathrm{m})$. The carrier gas was helium (flow $2 \mathrm{~mL} \mathrm{~min}-1$, split ratio 10: 1 , the volume of sampling $1 \mu \mathrm{L}$ ). The injection and ionization temperature was set at $200{ }^{\circ} \mathrm{C}$. The standard curves of acetate, propionate and butyrate were established and the concentrations of these SCFAs ( $\mathrm{mmol} / \mathrm{kg}$ mouse feces) were calculated according to the standard curve.

Statistics. Statistical analysis was performed using GraphPad Prism 6.01 (GraphPad Software, Inc., San Diego, CA) unless otherwise specified. Data obtained from experiments are shown as means \pm s.e.m. Differences in body weight and OGTT were analyzed by the unpaired two-tailed Student's $t$-tests, and those with more than two groups were assessed with one-way ANOVA followed by Newman-Keuls post hoc tests. In the figures, the data with different superscript letters are significantly different based on post hoc ANOVA statistical analysis. Graph bars marked with different letters on top represented statistically significant results $(P<0.05)$ based on Newman-Keuls post hoc one-way ANOVA analysis, whereas bars labeled with the same letter correspond to results that show no statistically significant differences. In the case, whereas two letters are present on top 
of the bar, each letter should be compared separately with the letters of other bars to determine whether the results show statistically significant differences.

Accession codes. Both sequencing data for the 16S rRNA and ITS sequences have been deposited in the NCBl's Sequence Read Archive database under BioProject ID No PRJNA506283. The 18S rRNA of E. cristaum CB10001 has been deposited in GenBank (MK371789).

\section{References}

1. Puhl, R. M. , \& Heuer, C. A. The stigma of obesity: a review and update. Obesity 17, 941-964 (2012).

2. Yoshimoto, S. et al. Obesity-induced gut microbial metabolite promotes liver cancer through senescence secretome. Nature 499, 97-101 (2013).

3. Osborn, O. \& Olefsky, J. M. The cellular and signaling networks linking the immune system and metabolism in disease. Nat. Med. 18, 363-374 (2012).

4. ElSayed Moustafa, J. S. \& Froguel, P. From obesity genetics to the future of personalized obesity therapy. Nat. Rev. Endocrinol. 9, 402-413 (2013).

5. Abarca-Gómez, L. et al. Worldwide trends in body-mass index, underweight, overweight, and obesity from 1975 to 2016: a pooled analysis of 2416 populationbased measurement studies in 1289 million children, adolescents, and adults. Lancet 390, 2627-2642 (2017).

6. Collaborators, G. O. et al. Health effects of overweight and obesity in 195 countries over 25 years. N. Engl. J. Med. 377, 13-27 (2017).

7. Saltiel, A. R. New therapeutic approaches for the treatment of obesity. Sci. Transl. Med. 8, 323rv322 (2016).

8. Srivastava, G. \& Apovian, C. M. Current pharmacotherapy for obesity. Nat. Rev. Endocrinol. 14, 12-24 (2017).

9. Cani, P. D., Bibiloni, R., Knauf, C., Waget, A. \& Neyrinck, A. M. Changes in gut microbiota control metabolic endotoxemia-induced inflammation in high-fat dietinduced obesity and diabetes in mice. Diabetes 57, 1470-81 (2008)

10. Gentile, C. L. \& Weir, T.L. The gut microbiota at the intersection of diet and human health. Science 362, 776-780 (2018).

11. Cani, P. D., Bibiloni, R., Knauf, C., Waget, A. \& Neyrinck, A. M. Changes in gut microbiota control metabolic endotoxemia-induced inflammation in high-fat dietinduced obesity and diabetes in mice. Diabetes 57, 1470-81 (2008).

12. Chang, C. J. et al. Ganoderma lucidum reduces obesity in mice by modulating the composition of the gut microbiota. Nat. Commun. 8, 16130-16147 (2017). 
13. Compare, D., Rocco, A., Sanduzzi, Z. M. \& Nardone, G. The gut bacteria-driven obesity development. Dig. Dis. 34, 221-229 (2016).

14. Villanuevamillán, M. J., Pérezmatute, P. \& Oteo, J. A. Gut microbiota: a key player in health and disease. A review focused on obesity. J. Physiol. Biochem. 71, 509525 (2015).

15. Everard, A. et al. Cross-talk between Akkermansia muciniphila and intestinal epithelium controls diet-induced obesity. Proc. Nat Acad. Sci. USA. 110, 9066-9071 (2013).

16. Sun, C. Q., O'Connor, C. J., Turner, S. J., Lewis, G. D. \& Stanley, R. A. The effect of $\mathrm{pH}$ on the inhibition of bacterial growth by physiological concentrations of butyric acid: implications for neonates fed on suckled milk. Chem-biol.Interact. 113, 117131 (1998).

17. Duncan, S. H., Louis, P., Thomson, J. M. \& Flint, H. J. The role of $\mathrm{pH}$ in determining the species composition of the human colonic microbiota. Environ. Microbiol. 11, 2112-2122 (2009).

18. Sonnenburg, E. D. et al. Specificity of polysaccharide use in intestinal Bacteroides species determines diet-induced microbiota alterations. Cell. 141, 1241-1252 (2010).

19. Smith, P. M. et al. The microbial metabolites, short-chain fatty acids, regulate colonic Treg cell homeostasis. Science 341, 569-573 (2013).

20. Ivey, M., Massel, M. \& Phister, T. G. Microbial interactions in food fermentations. Annu. Rev. Food. Sci. T. 4, 141-162 (2012).

21. Marco, M. L. \& Golomb B. L. Fermented foods, Lactobacillus, and health: Lactobacillus bacteria serve as a gateway for understanding transitory hostmicrobe interactions in the digestive tract. Microbe 11, 349-354 (2016).

22. Mcgovern, P. E. et al. Fermented beverages of pre- and proto-historic China. Proc. Natl Acad. Sci. USA. 101, 17593-17598 (2004).

23. Ge, Y. et al. Comparative genomic and transcriptomic analyses of the Fuzhuan brick tea-fermentation fungus Aspergillus cristatus. BMC Genomics 17, 428-441 (2016).

24. Meng, T., Zeng, X. \& Zou, X. Study on the birth time of Fuzhuan brick tea. J. Fujian. tea. (in Chinese) 37, 140-142 (2015).

25. Foster, M. T. et al. Fuzhuan tea consumption imparts hepatoprotective effects and alters intestinal microbiota in high saturated fat diet-fed rats. Mol. Nut. Food. Res. 60, 1213-1220 (2016).

26. Chen, G. et al. Fuzhuan brick tea polysaccharides attenuate metabolic syndrome in high-fat diet induced mice in association with modulation in the gut microbiota. J. Agric. Food. Chem. 66, 2783-2795 (2018).

27. Fu, D. et al. Fermented Camellia sinensis, Fu zhuan tea, regulates hyperlipidemia and transcription factors involved in lipid catabolism. Food. Res. Int. 44, 2999-3005 (2011).

28. Lee, D. M. et al. Fuzhuan tea reverses arterial stiffening after modest weight gain in mice. Nutrition 33, 266-270 (2017).

29. Chen, G. et al. Kudingcha and Fuzhuan brick tea prevent obesity and modulate gut 
microbiota in high-fat diet fed mice. Mol. Nutr. Food. Res. 62, e1700485 (2018).

30. Li, Q. et al. Fungal community succession and major components change during manufacturing process of Fu brick tea. Sci. Rep-UK. 7, 6947-6954 (2017).

31. Cani, P. D. et al. Changes in gut microbiota control inflammation in obese mice through a mechanism involving GLP-2-driven improvement of gut permeability. Gut 58, 1091-1103 (2009).

32. $\mathrm{Ng}, \mathrm{M}$. et al. Global, regional, and national prevalence of overweight and obesity in children and adults during 1980-2013: a systematic analysis for the Global Burden of Disease Study 2013. Lancet 384, 766-781 (2014).

33. Jiang, T. T. et al. Commensal fungi recapitulate the protective benefits of intestinal bacteria. Cell. Host. Microbe. 22, 809-816 (2017).

34. Limon, J. J., Skalski, J. H. \& Underhill, D. M. Commensal fungi in health and disease. Cell. Host. Microbe. 22, 156-165 (2017).

35. Turnbaugh, P. J., Ley, R. E., Mahowald, M. A., Magrini, V. \& Mardis, E. R. An obesity-associated gut microbiome with increased capacity for energy harvest. Nature 444, 1027-1031 (2006).

36. Goodman, A. L. et al. Extensive personal human gut microbiota culture collections characterized and manipulated in gnotobiotic mice. Proc. Natl Acad. Sci. USA. 108, 6252-6257 (2011).

37. Serena, C. et al. Elevated circulating levels of succinate in human obesity are linked to specific gut microbiota. ISME J. 12, 1642-1657 (2018).

38. Liu, G., Bei, J., Liang, L., Yu, G., Li, L. \& Li, Q. Stachyose improves inflammation through modulating gut microbiota of high-fat diet/streptozotocin induced type 2 diabetes in rats. Mol. Nutr. Food. Res. 62, e1700954 (2018).

39. Langille, M. G. I. et al. Predictive functional profiling of microbial communities using 16S rRNA marker gene sequences. Nat. Biotechnol.31, 814-821 (2013).

40. Schwiertz, A. et al. Microbiota and SCFA in lean and overweight healthy subjects. Obesity 18, 190-195 (2012).

41. Turnbaugh, P. J. et al. A core gut microbiome in obese and lean twins. Nature 457, 480-4 (2009).

42. Anhê, F. F. et al. Treatment with camu camu (Myrciaria dubia) prevents obesity by altering the gut microbiota and increasing energy expenditure in diet-induced obese mice. Gut 9, 315565-315580 (2018).

43. $\mathrm{Xu}, \mathrm{P}$. et al. Melatonin prevents obesity through modulation of gut microbiota in mice. J. Pineal. Res. 62, e12399 (2017).

44. Behnsen, J., Deriu, E., Sassone-Corsi, M. \& Raffatellu M. Probiotics: properties, examples, and specific applications. CSH. Perspect. Med. 3, a010074 (2013).

45. Cani, P. D. et al. Selective increases of bifidobacteria in gut microflora improve high-fat-diet-induced diabetes in mice through a mechanism associated with endotoxaemia. Diabetologia 50, 2374-2383 (2007).

46. Woting, A. \& Blaut, M. The intestinal microbiota in metabolic disease. Nutrients 8 , 202-220 (2016).

47. Collado, M. C., Isolauri, E., Laitinen, K. \& Salminen S. Distinct composition of gut microbiota during pregnancy in overweight and normal-weight women. Am. J. Clin 
Nutr. 88, 894-899 (2008).

48. KalliomãKi, M., Collado, M. C., Salminen, S. \& Isolauri, E. Early differences in fecal microbiota composition in children may predict overweight. Am. J. Clin Nutr. 87, 534-538 (2008).

49. $\mathrm{Wu}, \mathrm{X}$. et al. Molecular characterisation of the faecal microbiota in patients with type II diabetes. Curr. Microbiol. 61, 69-78 (2010).

50. Ottman, N., Geerlings, S. Y., Aalvink, S., de Vos, W. M. \& Belzer, C. Action and function of Akkermansia muciniphila in microbiome ecology, health and disease. Best. Pract. Res. Clin. Gastroenterol. 31, 637-642 (2017).

51. Derrien, M., Belzer, C. \& Vos, W. M. D. Akkermansia muciniphila and its role in regulating host functions. Microb. Pathogenesis. 106, 171-181 (2017).

52. Qin, J. et al. A metagenome-wide association study of gut microbiota in type 2 diabetes. Nature 490, 55-60 (2012).

53. Sawicki, C. M., Livingston, K. A., Obin, M., Roberts, S. B. \& Mei, C. Dietary fiber and the human gut Microbiota: application of evidence mapping methodology. Nutrients 9, 125-146 (2017).

54. Zhao, L. et al. Gut bacteria selectively promoted by dietary fibers alleviate type 2 diabetes. Science 359, 1151-1156 (2018).

55. Poul, E. L. et al. Functional Characterization of human receptors for short chain fatty acids and their role in polymorphonuclear cell activation. J. Bio. Chem. 278, 25481-25489 (2003).

56. Cox, M. A. et al. Short-chain fatty acids act as anti-inflammatory mediators by regulating prostaglandin E2 and cytokines. World. J. Gastroentero. 15, 5549-5557 (2009).

57. Canfora, E. E., Jocken, J. W. \& Blaak, E. E. Short-chain fatty acids in control of body weight and insulin sensitivity. Nat. Rev. Endocrinol. 11, 577-591 (2015).

58. Caporaso, J. G. et al. QIIME allows analysis of high-throughput community sequencing data. Nat. Methods 7, 335-336 (2010).

59. Costabile, A. et al. Whole-grain wheat breakfast cereal has a prebiotic effect on the human gut microbiota: a double-blind, placebo-controlled, crossover study. Brit. J. Nutr. 99, 110-120 (2008).

\section{Acknowledgements}

The authors thank the Center for Advanced Research for the GCMS and Minerals processing and bioengineering for Illumina MiSeq platform in Central South University. This work was supported in parts by NSFC grant 81473124 (to Y. H.), the Chinese Ministry of Education 111 Project B0803420 (to Y. D.).

\section{Author contributions}

Y.H. and Y.D. conceived the project; Y.H., Y.D. and D.K. designed the experiments; D.K. and M.S. performed the experiments; Y.H., and D.K. analyzed the results and wrote the manuscript with inputs from all co-authors.

\section{Competing interests.}


The authors declare no competing financial interests.

\section{Materials \& Correspondence}

All the correspondence and material requests should be addressed to jonghuang@csu.edu.cn (Y.H.) or ywduan66@sina.com (Y.D.).

\section{Animal protocols}

All animal protocols were approved by the Animal Care and Use Committee of Central South University. All experimental procedures complied with the Guide for the Care and Use of Laboratory Animals (1996).

\section{Additional information}

Supplementary information is available online.

\section{Competing interests}

There are no conflicts to declare. 